Revista Universo Contábil, ISSN 1809-3337

doi:10.4270/ruc.2012103

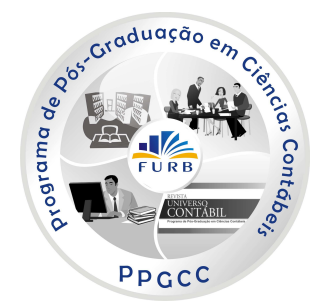

\title{
A ANÁLISE FINANCEIRA FUNDAMENTALISTA NA PREVISÃO DE MELHORES E PIORES ALTERNATIVAS DE INVESTIMENTO'
}

\author{
FUNDAMENTAL FINANCIAL ANALYSIS IN PREDICTING BEST AND WORST \\ INVESTMENT ALTERNATIVES
}

\section{EL ANALISIS FINANCIERO FUNDAMENTALISTA EN LA PREVISION DE MEJORES Y PEORES OPORTUNIDADES DE INVERSIÓN}

\author{
Adilson de Lima Tavares \\ Doutor em Ciências Contábeis pelo Programa Multiinstitucional e Inter-regional \\ de Pós-Graduação em Ciências Contábeis (UnB/UFPB/UFRN) \\ Endereço: Rua Jacarandá, 374 - Nova Parnamirim \\ CEP: 59152-210 - Parnamirim/RN - Brasil \\ E-mail: adilson.tavares@ufrnet.br \\ Telefone: (84) 3608-4158 \\ César Augusto Tibúrcio Silva \\ Doutor em Controladoria e Contabilidade pela FEA/USP \\ Professor do Programa Multiinstitucional e Inter-Regional de Pós-Graduação \\ em Ciências Contábeis (UnB/UFPB/UFRN) \\ Endereço: Campus Universitário Darcy Ribeiro, Prédio da FACE, Asa Norte \\ CEP: 70910-900 - Brasília/DF - Brasil \\ E-mail: cesartiburcio@unb.br \\ Telefone: (61) 3307-2345
}

\section{RESUMO}

Este artigo investiga se a Análise Financeira Fundamentalista (AFF) é capaz de segregar grupos de melhores e piores alternativas de investimentos a partir da previsão de variações do valor de mercado das empresas não-financeiras listadas na Bolsa de Valores de São Paulo (BOVESPA). As amostras do estudo correspondem a 2/3 das empresas com dados disponíveis no banco de dados da Economática nos anos de 2005, 2006 e 2007, e estão divididas em dois grupos: Perdedoras e Vencedoras. Foram selecionados 23 Índices Econômico-Financeiros (IEF) tradicionais, comumente utilizados por analistas e pesquisadores nas avaliações de oportunidades de investimento. O tratamento econométrico dos dados foi realizado com o uso

\footnotetext{
1 Artigo recebido em 04.04.2011. Revisado por pares em 21.07.2011. Reformulado em 18.09.2011. Recomendado para publicação em 21.09.2011 por Ilse Maria Beuren (Editora). Publicado em 31.01.2012. Organização responsável pelo periódico: FURB.
} 
da Regra do Qui-quadrado Mínimo e da Análise Discriminante. Constatou-se que um número bastante reduzido de IEF apresentou significância estatística à diferenciação entre as empresas integrantes das amostras. Apesar disso, as classificações corretamente previstas nos três exercícios sociais alcançaram níveis satisfatórios, indicando que o uso da AFF contribui para melhorar os resultados das decisões de investimentos. Conclui-se que é possível aceitar a hipótese de que a AFF é capaz de prever variações de valor da empresa, contribuindo para a escolha entre melhores e piores alternativas de investimentos no mercado de ações brasileiro.

Palavras-chave: Análise fundamentalista. Índices econômico-financeiros. Valor da empresa.

\section{ABSTRACT}

This paper investigates whether the Fundamental Financial Analysis (FFA) is able to segregate groups of best and worst investment alternatives from the prediction of changes in market-value of non-financial companies listed in Bolsa de Valores de São Paulo (BOVESPA). The study samples correspond to $2 / 3$ of the non-financial companies with available data in the Economatica database in the years 2005, 2006 and 2007, and they are divided in two groups: Losers and Winners. 23 traditional Financial-Economic Ratios (FER), commonly used by analysts and researchers in assessing investment opportunities, were selected. The econometric treatment of data was performed using the Minimum Chi-Square Rule and the Discriminant Analysis. It was found that a very small number of FER showed statistic significance to the differentiation between the companies of the samples. Nevertheless, the classifications predicted correctly in the three years reached satisfactory levels, indicating that the use of FFA contributes to improve the performance of investment decisions. It is concluded that it is possible to accept the hypothesis that FFA is able to predict changes in company value, contributing to the choice between better and worse investment alternatives in the Brazilian Stock Market.

Keyworks: Fundamental analysis. Financial-economic ratios. Company value.

\section{RESUMEN}

Este artículo investiga si el análisis financiero fundamentalista $(A F F)$ es capaz de apartar grupos de mejores y peores de inversión a partir de previsión de variaciones de valor de mercado de las empresas no-financieras listada en la Bolsa de Valores de São Paulo (BOVESPA). Las muestra de este estudio corresponden a un 2/3 de las empresas con datos disponibles en el banco de datos de la Economática en los años 2005,2006 y 2007. Estos datos están divididos en dos grupos: perdedoras y vencedoras. Fueron seleccionados 23 índices económico-financieros (IEF) tradicionales que suelen ser usados por analistas e investigadores en las evaluaciones de oportunidades de inversión. El tratamiento econométrico de los datos fue realizado con el uso de la regla Chi-cuadrado Mínimo y del Análisis Discriminante. Se ha constatado que una cantidad bastante reducida de IEF ha presentado significado estadístico a la diferencia entre las empresas integrantes de las muestras. Además de eso, las clasificaciones correctamente previstas en los tres ejercicios sociales alcanzan niveles satisfactorios, indicando que el uso de la AFF contribuye para mejorar los resultados de las decisiones de inversión. Se concluye que es posible aceptar la hipótesis de que la AFF es capaz de prever variaciones de valor de la empresa, contribuyendo para la elección entre las mejores y peores posibilidades de inversión en al mercado brasileño.

Palabras-clave: Análisis fundamentalista. Índices económico-financieros. Valor de la empresa. 


\section{INTRODUÇÃO}

A Análise Fundamentalista (AF) e a análise técnica são os dois tipos de análise comumente destacados na literatura para avaliação e escolha entre alternativas de investimento. A primeira baseia-se em informações extraídas das demonstrações contábeis, além da análise do mercado, do panorama econômico e de projeções de resultados. A segunda, também denominada análise gráfica, tem por base dados históricos relativos a preços e volumes dos ativos em períodos prévios, a partir dos quais o investidor analisará as tendências para decidir entre as alternativas de investimento.

Embora o processo de análise fundamentalista envolva estudos acerca das estratégias da empresa, da qualidade da contabilidade, dos dados financeiros e das projeções de resultados, esta pesquisa concentrará esforços na análise financeira, que utiliza dados financeiros, comumente na forma de índices, para sumariar as performances das empresas.

A Análise Financeira Fundamentalista (AFF), foco principal desta pesquisa, não é uma metodologia que tem aceitação unânime entre analistas e pesquisadores quanto à sua eficácia no auxílio à tomada de decisão sobre investimentos. No entanto, há estudos em vários países, tais como Japão (CHAN; HAMAO; LAKONISHOK, 1991), Estados Unidos (PIOTROSKI, 2000), Brasil (LOPES; GALDI, 2006) e Reino Unido (CLUBB; NAFFI, 2007), que têm apresentado resultados favoráveis ao uso de dados fundamentalistas na seleção entre alternativas de investimentos.

Considerando que o mercado de ações no Brasil tem se desenvolvido de forma mais vigorosa, e presumindo-se que, por esta razão, os analistas precisam ter disponíveis ferramentas que minimizem os riscos nas tomadas de decisões, surge a seguinte questão de pesquisa: A análise financeira fundamentalista é capaz de segregar grupos de melhores e piores alternativas de investimentos a partir da previsão de variações de valor das empresas?

A hipótese levantada neste estudo e de que a AFF é capaz de prever variações de valor da empresa, contribuindo para a escolha entre melhores e piores alternativas de investimentos no mercado de ações brasileiro. Espera-se que o estudo possa contribuir para uma reflexão acerca da adequabilidade das metodologias atualmente utilizadas, no mercado brasileiro de ações, para avaliação e escolha entre alternativas de investimentos.

Além desta introdução, este estudo compõe-se de mais cinco seções. As seções 2 e 3, ao tratarem da Análise Fundamentalista e de Índices Econômico-Financeiros, buscam fornecer a fundamentação teórica do artigo. A metodologia é apresentada de forma detalhada na seção 4, e os resultados e análises da pesquisa empírica constam na seção 5 . A seção 6 busca responder à questão formulada nesta introdução e apresenta as considerações finais.

\section{ANÁLISE FUNDAMENTALISTA (AF)}

A AF procura identificar as causas que explicam as variações nos preços das ações (LEMES JÚNIOR; RIGO; CHEROBIM, 2002), utilizando as demonstrações contábeis como uma de suas principais fontes informativas, das quais são extraídas informações sobre a liquidez, a rentabilidade, o endividamento, os níveis de atividade operacional, dentre outras. Tais informações são utilizadas para previsão de resultados, de oferta e demanda, da força da indústria, da capacidade gerencial e de outras questões que afetam o valor de mercado e o potencial de crescimento de uma ação (THOMSETT, 1998). Ao disponibilizar esses dados para análise, a contabilidade desempenha o papel de contestadora de crenças especulativas, buscando ancorar o investidor aos fundamentos da empresa (PENMAN, 2003).

A análise focada nos principais fundamentos econômicos e financeiros de uma empresa adota a hipótese da existência de um valor intrínseco para cada ativo (FOSTER, 1986; ASSAF NETO, 2007). O confronto entre o preço de mercado da ação e o seu valor intrínseco propicia ao analista uma visão do potencial de geração ou destruição de valor 
daquele título (QUIRIN; BERRY; O’BRYAN, 2000), o que subsidiará a tomada de decisões do investidor.

Kothari (2001) defende que o foco principal da análise fundamentalista repousa sobre a avaliação de ativos com o objetivo de identificar títulos malprecificados, procedimento esse que se tornou popular desde a publicação do livro Security Analysis, em 1934, por Graham e Dodd. Estes autores estão entre os primeiros a discutir formalmente a importância de fatores fundamentais no exercício da avaliação de ações (BETTMAN; SAULT; WELCH, 2006).

Esse processo de análise tem por premissa que as informações contábeis históricas têm valor preditivo (OLSON; MOSSMAN, 2003). Ele envolve o uso de informações correntes e passadas, extraídas das demonstrações contábeis, que em conjunto com dados macroeconômicos e do setor de atuação de cada empresa, propiciará a obtenção do valor da empresa (KOTHARI, 2001).

Na visão de Miltersteiner (2003), as informações mais relevantes nos campos setorial e macroeconômico são aquelas que se referem à concorrência, às políticas de crédito, fiscal e cambial que envolvem a entidade. Assevera ainda que quanto melhor for o conhecimento acerca desses aspectos, e das possíveis implicações que eles venham a ter sobre a atividade da empresa sob análise, melhor ela poderá ser avaliada.

Ao optar pelo investimento em ações de determinada companhia, o seu valor de mercado é um dado de relevante interesse do analista. Esse valor pode ser representado pelo produto da cotação de suas ações em bolsa numa determinada data e o número total de ações que integram o capital naquele mesmo momento (FERNÁNDEZ; REINOSO, 2002).

De acordo com Piotroski (2005), a premissa subjacente às estratégias de investimento baseadas na análise das demonstrações contábeis tradicionais é que os investidores sofisticados são capazes de selecionar oportunidades lucrativas pelo uso de informações históricas. Neste caso, infere-se que as informações acerca das situações econômicofinanceiras passadas e atuais das empresas são dotadas de grande valor e servem de inputs à análise prospectiva da rentabilidade de seus ativos.

O processo completo de análise fundamentalista, conforme Palepu, Healy e Bernard (2004), envolve:

a) análise das estratégias de negócios - para identificar os direcionadores-chave do lucro e os riscos do negócio, e avaliar o potencial de lucro em nível qualitativo.

b) análise contábil - tem por fim avaliar o grau em que a contabilidade captura a realidade subjacente aos negócios.

c) análise financeira - usa dados financeiros para avaliar as performances passada e atual da empresa, bem como sua sustentabilidade.

d) análise prospectiva - se constitui numa síntese da visão do analista, a partir da análise de negócios, da análise contábil e da análise financeira.

Neste estudo, a busca da resposta à questão de pesquisa está focada na Análise Financeira Fundamentalista (AFF) através de índices, obtidos a partir das demonstrações contábeis e informações de mercado.

\section{3 ÍNDICES ECONÔMICO-FINANCEIROS (IEF)}

A utilização de IEF no estudo da saúde econômico-financeira das empresas já ocorre há mais de um século. Conforme Myer (1976), há evidências de que em 1908 a medição quantitativa por meio de IEF já havia sido adotada pelos banqueiros, dado que as exigências à concessão de créditos levavam em consideração a liquidez, cuja medida considerava a razão entre o ativo a curto prazo e o passivo do tomador do crédito.

Por volta de 1913 começaram a surgir críticas à supremacia do quociente de liquidez em relação aos demais critérios de análise. Dentre os críticos estava Alexander Wall, que, em 1919, publicou um artigo intitulado Study of Credit Barometrics no Federal Reserve Bulletin, 
criticando os banqueiros que usavam apenas o índice de liquidez como base para suas tomadas de decisões e mostrando que era necessário considerar outras relações além de ativos e passivos correntes para ter um quadro completo da situação da empresa (MYER, 1976).

A análise por meio de IEF ainda é uma das técnicas mais largamente utilizadas. Os IEF são obtidos, principalmente, a partir de relações entre valores extraídos das demonstrações contábeis. O que o analista busca com esses IEF, conforme Lyra (2008), é medir, comparar e projetar desempenhos, quer sejam econômicos, financeiros ou patrimoniais e, para tanto, os indicadores utilizados devem conter os seguintes aspectos básicos: objetividade, mensurabilidade, compreensibilidade e comparabilidade.

Conforme Foster (1986), as principais motivações para a análise através de IEF são: a) realizar o controle por diferenças de tamanho na comparação entre firmas e ao longo do tempo; b) fazer com que os dados satisfaçam melhor as premissas subjacentes às ferramentas estatísticas, tais como a análise de regressão; c) provar uma teoria na qual um índice é a variável de interesse; e d) explorar uma regularidade empírica observada entre um índice financeiro e a estimação ou predição de uma variável de interesse (ex: o risco de um título ou a probabilidade de uma firma declarar falência).

Lyra (2008) destaca críticas básicas aos indicadores comumente utilizados nas análises de desempenho de empresas, identificadas na revisão teórica de sua tese de doutorado. Adverte que: a) a liquidez obtida a partir de índices é uma relação estática do passado, e pode não mais refletir a capacidade financeira no momento da análise; b) há incompatibilidade na comparação de alguns itens relacionados aos índices de estrutura de capital; c) os índices de rentabilidade possuem itens extraídos da demonstração de resultado que não considera o custo do capital próprio; e 4) os cálculos dos índices de atividades realizados por usuários externos sofrem limitações, devido à falta de detalhamento das demonstrações contábeis.

Por sua vez, Gasparetto (2004) entende que a contabilidade se constitui num importante provedor de informações à avaliação do desempenho da empresa, e que, embora haja críticas à utilização de indicadores tradicionais nos modelos de avaliação desenvolvidos, eles têm-se revelado importantes nessa tarefa. Ainda segundo Gasparetto (2004), as críticas à utilização de IEF tradicionais não devem levar à substituição desses, como apregoam diversos autores, mas à complementação com outros indicadores, tais como os não-financeiros.

Neste estudo, são utilizados recursos econométricos para verificar a relação existente entre IEF tradicionais e a previsão de movimentos ascendentes ou descendentes nos valores de mercado das empresas.

\section{METODOLOGIA}

\subsection{Seleção das Amostras}

A população neste estudo é composta de empresas não-financeiras listadas na Bolsa de Valores de São Paulo (BOVESPA), nos exercícios de 2005, 2006 e 2007. Dados posteriores a esse período não são objeto de amostra em razão das mudanças em curso nas práticas contábeis, decorrentes do processo de convergência das normas brasileiras às normas internacionais de Contabilidade. Outro fator relevante na delimitação do período das amostras utilizadas decorre da instabilidade dos mercados financeiros de diversos países a partir de 2008, decorrente da Crise do Subprime. Portanto, como as informações contábeis divulgadas pelas companhias podem estar carregadas pelos efeitos da crise, e considerando a fase de transição do processo de convergência, foram evitados os exercícios de 2008 a 2010.

Para este estudo, foi realizado um levantamento de todas as empresas não-financeiras com sede no Brasil que constavam no banco de dados da Economática no período de 2005 a 2007. Dessas empresas, num total de 561, foram excluídas aquelas que tiveram seus registros cancelados na Bolsa de Valores de São Paulo e que ainda constavam no banco de dados. 
Os indicadores econômico-financeiros que têm por denominador o patrimônio líquido das empresas podem não ter uma interpretação óbvia quando estes apresentam valores negativos (FOSTER, 1986). Portanto, foram excluídas do processo de seleção da amostra todas as empresas que apresentaram tal situação patrimonial.

Para evitar informações enviesadas, outro aspecto avaliado foi a possível existência de fatos extraordinários ocorridos durante o exercício social que pudessem provocar elevação ou declínio das cotações de ações de forma artificial. Isto é, aumentos ou diminuições que não decorressem do desempenho econômico-financeiro e operacional nas atividades normais da companhia, tais como incorporações, fusões e ações especulativas do mercado.

Por fim, foram verificadas ainda algumas empresas que, embora atendessem aos demais pré-requisitos, não apresentaram quaisquer dados (ou a maioria deles) para as variáveis utilizadas no estudo. Após esse tratamento inicial, remanesceu uma população 195, 188 e 203 companhias para os exercícios de 2005, 2006 e 2007, respectivamente.

Tabela 1 - Dados quantitativos da seleção da amostra de cada exercício social

\begin{tabular}{|c|c|c|c|c|c|c|c|c|}
\hline \multirow[b]{2}{*}{ Ano } & \multirow{2}{*}{$\begin{array}{c}\text { Empresas } \\
\text { Não- } \\
\text { Financ. }\end{array}$} & \multicolumn{5}{|c|}{ Empresas Excluídas } & \multirow[b]{2}{*}{$\begin{array}{c}\text { Empresas } \\
\text { Remanescentes }\end{array}$} & \multirow[b]{2}{*}{ Amostras } \\
\hline & & $\begin{array}{c}\text { Registro } \\
\text { Cancelado } \\
\end{array}$ & $\begin{array}{l}\text { Sem Vr. } \\
\text { Mercado } \\
\end{array}$ & $\begin{array}{c}\text { PL } \\
\text { Negativo } \\
\end{array}$ & \begin{tabular}{|c|} 
Fatos \\
Extraord. \\
\end{tabular} & $\begin{array}{c}\text { Sem } \\
\text { Dados }\end{array}$ & & \\
\hline 2005 & 561 & 123 & 212 & 26 & 2 & 3 & 195 & 132 \\
\hline 2006 & 561 & 144 & 200 & 24 & 3 & 2 & 188 & 126 \\
\hline 2007 & 561 & 156 & 160 & 31 & 3 & 8 & 203 & 136 \\
\hline
\end{tabular}

Fonte: Dados da pesquisa.

Para a totalidade das empresas que atenderam às exigências iniciais, foram levantadas as variações percentuais dos valores de mercado entre o fechamento do exercício social precedente e o fechamento de cada exercício social em análise. Dispondo destas informações, as empresas foram classificadas em ordem crescente de geração de valor, tendo por referencial as variações relativas de valores de mercado.

Integraram a amostra de cada exercício social as empresas do primeiro terço (33,33\% da população), aquelas que obtiveram menor agregação de valor (Empresas Perdedoras), bem como as empresas do terceiro terço (outros $33,33 \%$ da população), que apresentaram maior agregação de valor (Empresas Vencedoras), o que corresponde a 2/3 das empresas da amostra disponível nos anos 2005 a 2007 (Tabela 1). A escolha dos grupos mais afastados da mediana da população visa a identificar perfis mais bem definidos das empresas integrantes de cada um deles, na busca de uma melhor discriminação das empresas a serem classificadas.

As amostras selecionadas necessitaram tratamentos por falta de dados e outliers (observações atípicas). A falta de dados (em média, 1,8\% dos dados de cada amostra), foi resolvida pela inserção de valores correspondentes à média aritmética das demais observações de cada grupo. Os outliers (em média, $0,58 \%$ dos dados de cada amostra) foram identificados a partir de técnicas de detecção univariada e multivariada, tomando por base a medida $D^{2}$ de Mahalanobis, e substituídas pela média aritmética das observações de cada grupo.

Após a seleção e tratamento de cada amostra anual, foi procedida a sua divisão em duas subamostras: de análise e de teste. As subamostras foram selecionadas aleatoriamente utilizando a rotina Random Number Generators do SPSS ${ }^{\circledR}$, numa proporção 60-40 entre análise e teste, respectivamente, resultando nos perfis apresentados na Tabela 2.

Tabela 2 - Segregação das amostras anuais em subamostras de análise e teste

\begin{tabular}{c|c|c|c}
\hline Ano & Amostra & Subamostra de Análise & Subamostra de Teste \\
\hline 2005 & 132 & 80 & 52 \\
\hline 2006 & 126 & 76 & 50 \\
\hline 2007 & 136 & 80 & 56 \\
\hline
\end{tabular}

Fonte: Dados da pesquisa. 


\subsection{Seleção dos Índices}

Para a seleção dos índices, foram utilizados resultados de três pesquisas: Anjos (2008), Lyra (2008) e Pinto e Hein (2008). Os IEF utilizados como variáveis independentes neste estudo, com as suas codificações e fórmulas de cálculo, estão relacionados na Tabela 3.

Tabela 3 - Códigos identificadores e fórmulas de cálculo dos IEF

\begin{tabular}{|c|c|c|}
\hline Código & Índice & Fórmula \\
\hline$X^{1}$ & Liquidez Imediata & $\frac{\text { Disponibilidades }}{P C}$ \\
\hline$X^{2}$ & Liquidez Corrente & $\frac{A C}{P C}$ \\
\hline$X^{3}$ & Liquidez Seca & $\frac{A C-\text { Estoques }}{P C}$ \\
\hline$X^{4}$ & Prazo de Pagamento Fornecedores & $\frac{\text { Fornecedores a pg }}{\text { Compras a Prazo }} \times 360$ \\
\hline$X^{5}$ & Prazo de Recebimentos & $\frac{\text { Duplicatas a receber }}{\text { Vendas a Prazo }} \times 360$ \\
\hline$X^{6}$ & Prazo de Estocagem & $\frac{\text { Est.de Merc./Produtos }}{\text { Custo Prod.Vendidos }} \times 360$ \\
\hline$X^{7}$ & Relação Capital de Terceiros/ Passivo Total & $\frac{\text { Exigível Total }}{\text { Passivo Total }}$ \\
\hline$X^{8}$ & Relação Capital de Terceiros/ Capital Próprio & $\frac{\text { Exigível Total }}{\text { Patrimônio Líquido }}$ \\
\hline$X^{9}$ & Dívida Financeira/Ativo Total & $\frac{\text { Emprést.e Financ. }}{\text { Total do Ativo }}$ \\
\hline$X^{10}$ & Dívida Financeira/Patrimônio Líquido & $\frac{\text { Emprést.e Financ. }}{\text { Patrimônio Líquido }}$ \\
\hline $\mathrm{X}^{11}$ & Dívida Financeira de C. Pz/Dívida Financeira Total & $\frac{\text { Emprést.e Financ. }(\text { C. Pz) }}{\text { Emprést.e Financ. }(\text { C.e L.Pz) }}$ \\
\hline$X^{12}$ & Lucro Operacional/Dívida Financeira & $\frac{\text { Lucro Operacional }}{\text { Emprést.e Financ. }}$ \\
\hline$X^{13}$ & Giro do Ativo & $\frac{\text { Receita Líq. Oper. }}{\text { Total do Ativo }}$ \\
\hline$X^{14}$ & Giro do Patrimônio Líquido & $\frac{\text { Receita Líq. Oper. }}{\text { Patrimônio Líquido }}$ \\
\hline$X^{15}$ & Margem Operacional & $\frac{\text { Lucro Operacional }}{\text { Receita Líq. Vendas }}$ \\
\hline$X^{16}$ & Margem Líquida & $\frac{\text { Lucro Líq. Exerc. }}{\text { Receita Líq.Vendas }}$ \\
\hline$X^{17}$ & Grau de Alavancagem Operacional & $\frac{\text { Lucro Bruto }}{\text { Lucro Oper. Próprio }}$ \\
\hline $\mathrm{X}^{18}$ & Retorno sobre o Ativo & $\frac{\text { Lucro Líq. Exerc. }}{\text { Ativo Total }}$ \\
\hline$X^{19}$ & Retorno sobre o Patrimônio Líquido & $\frac{\text { Lucro Líquido do Exercício }}{\text { Patrimônio Líquido }}$ \\
\hline$X^{20}$ & Margem Bruta & $\frac{\text { Lucro Bruto }}{\text { Receita Líq.Vendas }}$ \\
\hline$X^{21}$ & EBITDA por Ação & $\frac{\text { Lucro Oper. }+ \text { Deprec. }+ \text { Amort. }}{N^{o} \text { de Açôes }}$ \\
\hline$X^{22}$ & Lucro por Ação & $\frac{\text { Lucro Líq. Exercício }}{N^{o} \text { de Ações }}$ \\
\hline$X^{23}$ & Patrimônio Líquido por Ação & $\frac{P L}{N^{o} \text { de Ações }}$ \\
\hline
\end{tabular}

Fonte: Elaboração própria. 
No tratamento estatístico dos dados, buscou-se associar a cada empresa todos os índices de desempenho econômico-financeiro definidos acima.

\subsection{Aplicação da Regra do Qui-Quadrado Mínimo e da Análise Discriminante}

\subsubsection{Regra do Qui-Quadrado Mínimo}

Por esse método, valores menores do qui-quadrado $\chi^{2}$ sugerem que a empresa em análise aproxima-se mais de um grupo, ao passo que valores maiores para o $\chi^{2}$ significam que o perfil da empresa analisada desvia da média do grupo e, provavelmente, ela não poderia ser classificada no grupo com o qual está sendo comparada. Isto é conhecido como a "regra do qui-quadrado mínimo" (ASSAF NETO; SILVA, 2002).

A regra do qui-quadrado mínimo foi apresentada por Tatsuoka (1971) como uma metodologia que tem a propriedade de minimizar a probabilidade de erros de classificação quando uma determinada população apresenta distribuição normal multivariada com matrizes de dispersão iguais. Posteriormente, Besley e Osteryoung (1991) e Assaf Neto e Silva (2002) propuseram o uso da mesma metodologia para detectar probabilidade de insolvência de clientes na concessão de créditos.

Neste estudo, buscou-se antever se os valores das empresas terão aumento ou diminuição de valor a partir do estudo de seus fundamentos (expressos nos IEF oriundos de suas demonstrações contábeis). Para tanto, foram calculados dois valores de $\chi^{2}$ para cada empresa integrante da amostra: um em relação ao grupo das Empresas Perdedoras, e outro em relação ao grupo das Empresas Vencedoras. Os cálculos dos $\chi^{2}$ para cada empresa foram realizados a partir da aplicação das seguintes Equações:

$\chi_{i P}^{2}=x_{i P}{ }^{\prime} S_{T}^{-1} x_{i P}+\ln \left|S_{P}^{D}\right|$, para a empresa $i$, em relação ao grupo das Perdedoras, e

$\chi_{i V}^{2}=x_{i V}{ }^{\prime} S_{T}^{-1} x_{i V}+\ln \left|S_{V}^{D}\right|$, para a empresa $i$, em relação ao grupo das Vencedoras.

Onde: $S_{T}^{-1}$ é a inversa da estimativa da matriz de variância-covariância das variáveis da população; $x_{i P}{ }^{\prime}$ e $x_{i V}{ }^{\prime}$ são os vetores linhas dos desvios das variáveis da empresa $i$ da subamostra de teste em relação aos valores médios das variáveis dos grupos Empresas Perdedoras e Empresas Vencedoras da subamostra de análise; $x_{i P}$ e $x_{i V}$ são as transpostas daqueles vetores; e $\ln \left|S_{P}^{D}\right|$ e $\ln \left|S_{V}^{D}\right|$ os logaritmos naturais das determinantes das matrizes de dispersão para Perdedoras e Vencedoras.

O modelo é testado nos três exercícios sociais: 2005, 2006 e 2007. A metodologia será considerada eficaz para este estudo se o nível de similaridade entre a classificação prévia e aquela encontrada a partir do cálculo do $\chi^{2}$ for estatisticamente significativo.

Às mesmas subamostras de análise e teste foi aplicada a Análise Discriminante, a fim de dar mais robustez aos resultados alcançados no primeiro tratamento estatístico.

\subsubsection{Análise Discriminante}

A Análise Discriminante determina uma variável estatística discriminante, também denominada função discriminante, que combina duas ou mais variáveis independentes que melhor discriminarão entre os objetos (pessoas, empresas etc.) nos grupos definidos a priori (HAIR et al., 2009). A função assume a seguinte forma:

$$
Z_{j k}=a+W_{1} X_{1 k}+W_{2} X_{2 k}+\ldots+W_{n} X_{n k}+\varepsilon_{j k}
$$

Onde: $Z_{j k}=$ escore $Z$ discriminante da função discriminante $j$ para o objeto $k ; a=$ intercepto; $W_{i}=$ peso discriminante para a variável independente $i ; X_{i k}=$ variável independente $i$ para o objeto $k$; e $\varepsilon_{j k}=$ termo de erro estocástico. 
A questão de pesquisa deste estudo requer a segregação dos componentes da amostra em dois grupos, ou seja, uma classificação exaustiva e dicotômica entre Empresas Perdedoras e Empresas Vencedoras a partir da análise das variáveis independentes (IEF) extraídas dos demonstrativos contábeis das empresas no exercício social anterior. Portanto, entende-se que a aplicação da análise discriminante simples constitui um tratamento estatístico adequado à solução do problema de pesquisa.

Destaca-se como de suma importância, para o tratamento estatístico com o uso da análise discriminante, a atenção aos seguintes pressupostos, a fim de que possa ser conferida robustez aos resultados obtidos (HAIR et al., 2009; MÁRIO, 2007): Normalidade multivariada, Linearidade, Ausência de outliers, Ausência de multicolinearidade e Homogeneidade das matrizes de variâncias-covariâncias.

\section{RESULTADOS E ANÁLISES}

\subsection{Testes dos Pressupostos da Regra do Qui-quadrado Mínimo e Análise Discriminante}

Os resultados dos testes estatísticos dos pressupostos da Regra do Qui-quadrado Mínimo e da Análise Discriminante para os dados das amostras foram satisfatórios. Destacase apenas que, para a amostra do ano 2006, o pressuposto da homogeneidade das matrizes de variâncias-covariâncias não foi atendido, dado que houve significância suficiente (Sig. 0,000) para rejeitar tal hipótese. Foi utilizado o teste Box's $M$, cuja finalidade é avaliar a similaridade das matrizes de dispersão das variáveis independentes entre os grupos (HAIR et al., 2009).

\subsection{Resultados da Regra do Qui-quadrado Mínimo}

No Painel A da Tabela 4 são apresentadas as classificações decorrentes da aplicação desta metodologia (Resultado do Teste) e as classificações efetivas (Resultado Real), verificadas ao final de cada exercício social. Nestes primeiros cálculos, foram utilizadas todas as variáveis do estudo após a exclusão daquelas que apresentaram alta multicolinearidade $\left(\mathrm{X}^{15}\right.$ em 2006 e $X^{2}, X^{14}$ e $X^{15}$ em 2007).

Pode-se observar que o nível de acertos é menor no ano de 2006 - exatamente o exercício social que não apresenta homogeneidade entre as matrizes de dispersão dos grupos. Para os demais anos, os níveis de acertos foram sensivelmente melhores. Vale ressaltar que as empresas apresentam variáveis com características muito parecidas entre os grupos, contruibuindo para que os qui-quadrados calculados em relação a Perdedoras e Vencedoras tenham diferenças muito pequenas e aumente a probabilidade de erro de classificação.

Tabela 4 - Matriz de Classificação pela Regra do Qui-quadrado Mínimo

\begin{tabular}{|c|c|c|c|c|c|c|c|c|c|c|}
\hline \multicolumn{11}{|c|}{ Painel A - Uso de todas as variáveis } \\
\hline \multicolumn{2}{|r|}{ Exercícios } & \multicolumn{3}{|c|}{2005} & \multicolumn{3}{|c|}{2006} & \multicolumn{3}{|c|}{2007} \\
\hline & & \multicolumn{3}{|c|}{ Resultado do Teste } & \multicolumn{3}{|c|}{ Resultado do Teste } & \multicolumn{3}{|c|}{ Resultado do Teste } \\
\hline & & Perd. & Venc. & Total & Perd. & Venc. & Total & Perd. & Venc. & Total \\
\hline \multirow{3}{*}{$\underset{\mathbb{J}}{\widetilde{\Xi}}$} & Perdedoras & 16 & 10 & 26 & 18 & 7 & 25 & 19 & 9 & 28 \\
\hline & Vencedoras & 5 & 21 & 26 & 13 & 12 & 25 & 9 & 19 & 28 \\
\hline & Total & 21 & 31 & 52 & 31 & 19 & 50 & 28 & 28 & 56 \\
\hline \multicolumn{2}{|c|}{ Níveis de Acertos } & \multicolumn{3}{|c|}{$\mathbf{7 1 . 2 \%}$} & \multicolumn{3}{|c|}{$60,0 \%$} & \multicolumn{3}{|c|}{$67.9 \%$} \\
\hline \multicolumn{11}{|c|}{ Painel B - Uso das variáveis com maior poder discriminante } \\
\hline \multirow{3}{*}{\multicolumn{2}{|c|}{ Exercícios }} & \multicolumn{3}{|c|}{2005} & \multicolumn{3}{|c|}{2006} & \multicolumn{3}{|c|}{2007} \\
\hline & & \multicolumn{3}{|c|}{ Resultado do Teste } & \multicolumn{3}{|c|}{ Resultado do Teste } & \multicolumn{3}{|c|}{ Resultado do Teste } \\
\hline & & Perd. & Venc. & Total & Perd. & Venc. & Total & Perd. & Venc. & Total \\
\hline \multirow{3}{*}{$\underset{\mathscr{U}}{\mathbb{G}}$} & Perdedoras & 19 & 7 & 26 & 22 & 3 & 25 & 20 & 8 & 28 \\
\hline & Vencedoras & 8 & 18 & 26 & 20 & 5 & 25 & 14 & 14 & 28 \\
\hline & Total & 27 & 25 & 52 & 42 & 8 & 50 & 34 & 22 & 56 \\
\hline \multicolumn{2}{|c|}{ Níveis de Acertos } & \multicolumn{3}{|c|}{$71,2 \%$} & \multicolumn{3}{|c|}{$54,0 \%$} & \multicolumn{3}{|c|}{$60,7 \%$} \\
\hline
\end{tabular}

Fonte: Dados da pesquisa. 
Os resultados encontrados no Painel B da Tabela 4 decorrem de novos cálculos dos $\chi_{V}^{2}$ e $\chi_{P}^{2}$ (Tabela 5) para cada empresa, utilizando apenas as variáveis que apresentaram maior poder discriminante entre os grupos do estudo.

Tabela 5 - Qui-quadrados e $\mathrm{Z}$ discriminante para matrizes de variâncias-covariâncias das variáveis significantes

\begin{tabular}{|c|c|c|c|c|c|c|c|c|c|c|c|}
\hline \multicolumn{4}{|c|}{2005} & \multicolumn{4}{|c|}{2006} & \multicolumn{4}{|c|}{2007} \\
\hline Empresas & $\chi_{p}^{2}$ & $\chi_{v}^{2}$ & $Z_{i}$ & Empresas & $\chi_{p}^{2}$ & $\chi_{v}^{2}$ & $Z_{i}$ & Empresas & $\chi_{p}^{2}$ & $\chi_{v}^{2}$ & $Z_{i}$ \\
\hline Ienergia & 0,0108 & 0,0022 & 0,6907 & Kepler Weber & 33,8902 & 21,0794 & 2,0145 & Santanense & 0,0515 & 0,3001 & $-0,2006$ \\
\hline Dixie Toga & 0,0016 & 0,0095 & $-0,6287$ & Karsten & 17,9525 & 18,4233 & 0,4989 & OHL Brasil & 0,4623 & 0,0101 & 0,3649 \\
\hline Braskem & 0,0000 & 0,0032 & $-0,2593$ & Quattor Petr & 17,7761 & 18,9659 & $-0,117$ & Gol & 0,5716 & 1,3774 & $-0,6503$ \\
\hline Brasmotor & 0,0005 & 0,0064 & $-0,4704$ & Petroflex & 15,7472 & 18,8091 & $-0,4324$ & Marisol & 0,4915 & 1,8140 & $-1,0672$ \\
\hline V C P & 0,0177 & 0,0058 & 0,9546 & Celul Irani & 15,9149 & 18,9211 & $-0,4921$ & Gradiente & 2,6692 & 1,0649 & 1,2946 \\
\hline Pronor & 0,0008 & 0,0008 & 0,0046 & Met Duque & 17,7970 & 20,4528 & $-1,3415$ & Dasa & 0,3951 & 1,2125 & $-0,6597$ \\
\hline GPC Part & 0,0003 & 0,0016 & $-0,1009$ & Cedro & 15,8435 & 19,0239 & $-0,5978$ & Sao Carlos & 0,6142 & 1,4803 & $-0,6989$ \\
\hline Ferbasa & 0,0048 & 0,0002 & 0,374 & Hoteis Othon & 29,6442 & 20,7669 & 0,9999 & Metal Leve & 2,8240 & 1,4190 & 1,1337 \\
\hline Pettenati & 0,0021 & 0,0106 & $-0,6814$ & Petroq Uniao & 16,2372 & 19,4880 & $-0,9033$ & Cedro & 0,5281 & 0,0545 & 0,3822 \\
\hline Sultepa & 0,0000 & 0,0026 & $-0,2065$ & Gol & 16,8787 & 18,8333 & $-0,197$ & Abnote & 1,6040 & 2,8204 & $-0,9816$ \\
\hline Klabin S/A & 0,0065 & 0,0190 & $-0,9981$ & Mangels Indl & 15,8770 & 18,7612 & $-0,3599$ & Cemar & 1,0664 & 2,0703 & $-0,8101$ \\
\hline Whirlpool & 0,0005 & 0,0064 & $-0,4704$ & Renar & 16,4959 & 19,5978 & $-0,934$ & CCR Rod. & 0,6611 & 1,4088 & $-0,6034$ \\
\hline Mangels Indl & 0,0147 & 0,0317 & $-1,3675$ & Alfa Consorc & 16,2172 & 18,6618 & $-0,1928$ & Datasul & 1,6319 & 0,5785 & 0,8501 \\
\hline Mundial & 0,0000 & 0,0026 & $-0,2065$ & Santistextil & 15,7983 & 18,8416 & $-0,4526$ & Medial Saude & 2,6246 & 4,8158 & $-1,7682$ \\
\hline Copesul & 0,0005 & 0,0011 & $-0,0482$ & CEG & 18,0471 & 18,7390 & 0,1599 & Embratel Part & 0,0868 & 0,7570 & $-0,5409$ \\
\hline Lix da Cunha & 0,0016 & 0,0095 & $-0,6287$ & Alfa Holding & 16,4493 & 18,6570 & $-0,1354$ & Pet Manguinh & 2,7342 & 1,1732 & 1,2597 \\
\hline Joao Fortes & 0,0048 & 0,0159 & $-0,8925$ & Dohler & 16,2335 & 18,9048 & $-0,4143$ & CEG & 0,1736 & 0,5637 & $-0,3148$ \\
\hline Tecel S Jose & 0,0003 & 0,0055 & $-0,4176$ & CPFL Energia & 15,9440 & 18,6924 & $-0,2829$ & Sanepar & 0,7370 & 1,6151 & $-0,7086$ \\
\hline Drogasil & 0,0065 & 0,0190 & $-0,9981$ & Eletropaulo & 16,5658 & 18,4558 & 0,0845 & Vivo & 0,6181 & 1,8309 & $-0,9788$ \\
\hline Ripasa & 0,0001 & 0,0020 & $-0,1537$ & Cemig & 16,0254 & 18,5155 & $-0,0982$ & Rio Gde Ener & 0,5875 & 1,3333 & $-0,6019$ \\
\hline Millennium & 0,0008 & 0,0008 & 0,0046 & Mendes Jr & 24,8842 & 19,8805 & 0,6983 & Wlm Ind Com & 0,2135 & 0,8290 & $-0,4967$ \\
\hline Light S/A & 0,0012 & 0,0005 & 0,0574 & Embratel Part & 16,5550 & 18,7060 & $-0,1524$ & Sultepa & 6,0325 & 3,3366 & 2,1755 \\
\hline Pet Manguinh & 0,0003 & 0,0016 & $-0,1009$ & Itautec & 15,8386 & 19,0911 & $-0,659$ & Celpa & 0,7679 & 1,6376 & $-0,7018$ \\
\hline Acos Vill & 0,0209 & 0,0405 & $-1,5786$ & Ideiasnet & 16,1908 & 19,2427 & $-0,7203$ & Sabesp & 0,7281 & 1,5741 & $-0,6827$ \\
\hline Brasil T Par & 0,0005 & 0,0011 & $-0,0482$ & Ipiranga Dis & 17,2191 & 19,7663 & $-0,9148$ & Alpargatas & 0,8862 & 1,9649 & $-0,8704$ \\
\hline Fosfertil & 0,0033 & 0,0000 & 0,2685 & Trafo & 22,9978 & 18,9490 & 1,3945 & Petroflex & 0,3669 & 1,1324 & $-0,6177$ \\
\hline Ipiranga Pet & 0,0134 & 0,0297 & $-1,3147$ & Ampla Energ & 23,3781 & 19,2272 & 1,2171 & Lojas Renner & 3,8577 & 2,3943 & 1,1809 \\
\hline CEG & 0,0008 & 0,0008 & 0,0046 & Cemar & 16,7595 & 18,3376 & 0,2573 & AES Tiete & 0,8523 & 1,6822 & $-0,6697$ \\
\hline Vale R Doce & 0,0065 & 0,0006 & 0,4796 & Leco & 16,5499 & 19,7392 & $-1,047$ & Springer & 3,0345 & 1,3083 & 1,3929 \\
\hline $\begin{array}{l}\text { Perdigao S/A } \\
\text { ALL Amer }\end{array}$ & 0,0177 & 0,0058 & 0,9546 & Elektro & 16,1808 & 18,4294 & 0,0221 & Mont Aranha & 0,5234 & 1,3386 & $-0,6579$ \\
\hline Lat & 0,0001 & 0,0020 & $-0,1537$ & Bradespar & 18,9837 & 20,8434 & $-1,3942$ & Ferbasa & 1,5714 & 2,8329 & $-1,018$ \\
\hline D F Vasconc & 0,0027 & 0,0000 & 0,2157 & M G Poliest & 16,0582 & 18,9687 & $-0,5104$ & Trafo & 1,3716 & 0,5626 & 0,6529 \\
\hline $\begin{array}{l}\text { Bradespar } \\
\text { Telef. Data }\end{array}$ & 0,0086 & 0,0013 & 0,5851 & Fibam & 16,7095 & 18,6695 & $-0,0872$ & Am Inox Br & 0,1378 & 0,1715 & $-0,0272$ \\
\hline Hld & 0,0065 & 0,0006 & 0,4796 & Cemat & 16,3559 & 18,5233 & $-0,024$ & Vale R Doce & 1,1104 & 2,0848 & $-0,7863$ \\
\hline Plascar Part & 0,0000 & 0,0032 & $-0,2593$ & Sabesp & 17,2801 & 18,4051 & 0,334 & Light S/A & 0,7133 & 1,7593 & $-0,844$ \\
\hline Sao Carlos & 0,0086 & 0,0223 & $-1,1036$ & Weg & 16,2939 & 18,3983 & 0,0799 & Metisa & 0,2037 & 0,0422 & 0,1303 \\
\hline Ger Paranap & 0,0209 & 0,0077 & 1,0601 & Ger Paranap & 15,7360 & 18,7961 & $-0,4248$ & CEB & 2,8327 & 1,9206 & 0,736 \\
\hline Cemig & 0,0012 & 0,0005 & 0,0574 & Saraiva Livr & 16,3804 & 18,8447 & $-0,3254$ & Fibam & 0,2238 & 0,0306 & 0,1559 \\
\hline Cia Hering & 0,0027 & 0,0000 & 0,2157 & Eucatex & 34,7775 & 21,6799 & 1,4034 & Trevisa & 0,9393 & 2,2220 & $-1,0352$ \\
\hline AES Tiete & 0,0835 & 0,0539 & 2,3795 & Acos Vill & 19,4696 & 19,8283 & $-0,4978$ & Lix da Cunha & 4,4095 & 2,3430 & 1,6676 \\
\hline Coelba & 0,0056 & 0,0003 & 0,4268 & Caraiba Met & 16,1953 & 18,4641 & $-0,011$ & Magnesita & 0,7637 & 0,1618 & 0,4857 \\
\hline Petrobras & 0,0086 & 0,0013 & 0,5851 & Pettenati & 16,1355 & 18,6843 & $-0,232$ & Rede Energia & 0,2156 & 0,5896 & $-0,3018$ \\
\hline Itausa & 0,0770 & 0,0487 & 2,2739 & Localiza & 17,6817 & 18,9814 & $-0,1524$ & Quattor Petr & 0,6321 & 1,7959 & $-0,9392$ \\
\hline Celpe & 0,0000 & 0,0032 & $-0,2593$ & Duratex & 16,2366 & 18,5244 & $-0,0547$ & Iguacu Cafe & 2,1022 & 1,4208 & 0,5499 \\
\hline CPFL Energia & 0,0000 & 0,0032 & $-0,2593$ & Forjas Taurus & 20,0760 & 18,8752 & 0,6137 & Met Duque & 0,0018 & 0,3903 & $-0,3135$ \\
\hline Marisol & 0,0134 & 0,0035 & 0,7962 & Elekeiroz & 15,9583 & 19,2250 & $-0,7485$ & Renar & 0,2510 & 0,0940 & 0,1267 \\
\hline Rio Gde Ener & 0,0000 & 0,0032 & $-0,2593$ & Rede Energia & 19,7475 & 20,6149 & $-1,0675$ & Encorpar & 1,7722 & 2,8176 & $-0,8436$ \\
\hline Tim Part S/A & 0,0192 & 0,0067 & 1,0073 & Ienergia & 16,2266 & 19,2449 & $-0,6994$ & Eleva & 1,4829 & 0,5418 & 0,7595 \\
\hline Alpargatas & 0,1829 & 0,1376 & 3,6461 & Botucatu Tex & 30,5481 & 21,3502 & 0,6107 & Brasmotor & 1,7313 & 2,8123 & $-0,8724$ \\
\hline J B Duarte & 0,0008 & 0,0008 & 0,0046 & Melhor SP & 15,9843 & 18,6639 & $-0,2482$ & Cia Hering & 0,2577 & 0,9758 & $-0,5794$ \\
\hline Bahema & 0,0226 & 0,0429 & $-1,6314$ & & & & & Karsten & 1,5754 & 0,9721 & 0,4869 \\
\hline Rossi Resid & 0,0008 & 0,0008 & 0,0046 & & & & & Mendes Jr & 0,458 & 0,0182 & 0,3549 \\
\hline & & & & & & & & Drogasil & 0,4686 & 0,5909 & $-0,0986$ \\
\hline & & & & & & & & Tec Blumenau & 1,6988 & 2,8103 & $-0,8969$ \\
\hline & & & & & & & & Sam Industr & 4,1913 & 2,2737 & 1,5474 \\
\hline & & & & & & & & Tecnosolo & 3,2364 & 1,7324 & 1,2137 \\
\hline
\end{tabular}

Fonte: Dados da pesquisa.

Para a seleção das variáveis significativas foi utilizada a estatística Wilk's Lambda e o 
teste F-ANOVA. Como estas estatísticas testam a hipótese de igualdade entre as médias dos grupos, foram utilizadas as variáveis que apresentaram significância estatística menor que 0,05 , ou seja, que rejeitam a hipótese principal. Os testes resultaram significantes nas variáveis $X^{20}$ em 2005, $X^{5}$ e $X^{10}$ em 2006 e $X^{11}$ e $X^{17}$ em 2007.

Analisando os resultados do Painel $\mathrm{B}$, é possível verificar que o nível de acertos em 2005 é igual àquele do Painel $\mathrm{A}$, podendo-se inferir que as demais variáveis, além de $\mathrm{X}^{20}$, não têm influência significativa sobre o percentual total de acertos. Nos anos de 2006 e 2007, a situação não difere muito, uma vez que os níveis de acertos alcançados nesses exercícios, com apenas duas variáveis cada um, correspondem a $90 \%$ daquele obtido com 22 e 20 variáveis, respectivamente.

Um ponto interessante nesta fase do estudo é a análise da precisão de classificação demonstrada na matriz, através da comparação de seus resultados com algum padrão. O teste estatístico do poder discriminatório da matriz de classificação, quando comparada com o modelo de chances, é a estatística $Q$ de Press (HAIR et al., 2009).

Tabela 6 - Testes de Poder Discriminatório da Matriz de Classificação

\begin{tabular}{|c|c|c|c|}
\hline Exercícios & 2005 & 2006 & 2007 \\
\hline \multicolumn{4}{|c|}{ Validação Teórica $(Q$ de Press $): \chi^{2}$ crítico a $0,05=3,84$} \\
\hline Todas as variáveis & 9,31 & 2,00 & 7,14 \\
\hline Variáveis de Maior Poder Discriminante & 9,31 & 0,32 & 2,57 \\
\hline \multicolumn{4}{|c|}{ Validação Prática: Igual ou maior que $62,5 \%$} \\
\hline Todas as variáveis & $71,2 \%$ & $60,0 \%$ & $67,9 \%$ \\
\hline Variáveis de Maior Poder Discriminante & $71,2 \%$ & $54,0 \%$ & $60,7 \%$ \\
\hline
\end{tabular}

Fonte: Dados da pesquisa

Pelos resultados da estatística $Q$ de Press, constantes na Tabela 6, as classificações a partir de qui-quadrados calculados com todas as variáveis no exercício de 2006, a priori, não seriam consideradas estatisticamente melhores do que o critério de chances. Vale ressaltar, entretanto, que este teste é sensível ao tamanho da amostra (HAIR et al., 2009), de forma que a estatística de 2006 pode tornar-se significante, mesmo permanecendo com o mesmo percentual de acertos, se o número de empresas da subamostra de teste for aumentado. Este mesmo entendimento é aplicável ao $Q$ de Press para as classificações do ano de 2007, quando utilizadas apenas as variáveis de maior poder discriminatório.

Outra medida de significância da razão de sucesso da matriz de classificações é a validação prática. Hair et al. (2009) sugerem que a previsão de classificação seja, pelo menos, um quarto maior do que a obtida por chances. Neste caso, o nível de acertos deve ser igual ou superior a $62,5 \%(50 \% \times 1,25)$.

Quando a regra do qui-quadrado mínimo utiliza todas as variáveis, as classificações dos exercícios de 2005 e 2007 apresentam significância prática. Por sua vez, quando utilizadas apenas as variáveis de maior poder discriminante, apenas 2005 teria significância prática. Entretanto, como o percentual de acertos nas classificações de 2007 não difere muito daquele sugerido por Hair et al. (2009), acredita-se ser interessante considerá-lo válido.

\subsection{Resultados da Análise Discriminante}

A estimação da função discriminante foi realizada pelo método stepwise. Na Tabela 7 são apresentadas as variáveis que melhor discriminam as empresas entre os grupos de Perdedoras e Vencedoras. É possível verificar que foram selecionadas as mesmas variáveis utilizadas na avaliação pela Regra do Qui-quadrado Mínimo. 
Tabela 7 - Variáveis inseridas na função discriminante

\begin{tabular}{|c|c|c|c|c|c|c|c|c|c|c|}
\hline \multirow{3}{*}{ Exerc } & \multirow{3}{*}{ Passos } & \multirow{3}{*}{ Inseridas } & \multicolumn{8}{|c|}{ Wilks' Lambda } \\
\hline & & & \multirow{2}{*}{ Estatística } & \multirow{2}{*}{$g l 1$} & \multirow{2}{*}{$g l 2$} & \multirow{2}{*}{$g l 3$} & \multicolumn{4}{|c|}{ Exact $\mathbf{F}$} \\
\hline & & & & & & & Estatística & $g l 1$ & $g l 2$ & Sig. \\
\hline 2005 & 1 & $\mathrm{X} 20$ & ,936 & 1 & 1 & 78 & 5,377 & 1 & 78 &, 023 \\
\hline \multirow{2}{*}{2006} & 1 & $\mathrm{X} 5$ & ,891 & 1 & 1 & 74 & 9,053 & 1 & 74 & ,004 \\
\hline & 2 & $\mathrm{X} 10$ & ,839 & 2 & 1 & 74 & 6,988 & 2 & 73 & ,002 \\
\hline \multirow{2}{*}{2007} & 1 & $\mathrm{X} 11$ & ,947 & 1 & 1 & 78 & 4,356 & 1 & 78 & , 040 \\
\hline & 2 & $\mathrm{X} 17$ & ,896 & 2 & 1 & 78 & 4,492 & 2 & 77 & ,014 \\
\hline
\end{tabular}

Fonte: Dados da pesquisa - SPSS.

A primeira variável selecionada em cada exercício social para inclusão na função discriminante é aquela que, dentro da subamostra de análise, apresenta maior poder discriminante (menor valor para Wilks' Lambda e maior valor para F-ANOVA). A inclusão das demais variáveis ocorre desde que forneçam discriminação adicional estatisticamente significante entre os grupos, além daquelas diferenças já explicadas pelas variáveis na função discriminante.

$\mathrm{Na}$ Tabela 8 encontram-se os coeficientes das variáveis inseridas nas funções discriminantes e os valores das constantes obtidos a partir dos dados da subamostra de análise.

Tabela 8 - Coeficientes das funções discriminantes canônicas

\begin{tabular}{c|r|r|r|r|r}
\hline \multicolumn{2}{c|}{2005} & \multicolumn{2}{c|}{2006} & \multicolumn{2}{c}{$\mathbf{2 0 0 7}$} \\
\hline \multicolumn{2}{c|}{ Função } & \multicolumn{2}{c|}{ Função } & \multicolumn{2}{c}{ Função } \\
\hline$X^{20}$ & 5,277 & $X^{5}$ &, 020 & $X^{11}$ &, 024 \\
\hline Constante & $-1,631$ & $X^{10}$ &, 004 & $X^{17}$ &,- 085 \\
\hline & & Constante & $-1,698$ & Constante & -.872 \\
\hline
\end{tabular}

Coeficientes não padronizados

Fonte: Dados da pesquisa - SPSS

Utilizando as informações da Tabela 8 , as funções discriminantes para uma determinada empresa $k$, nos exercícios sociais de 2005, 2006 e 2007 podem ser apresentadas, respectivamente, com as seguintes disposições:

$$
\begin{aligned}
& Z_{k}=-1,631+5,277 X^{20}+\varepsilon_{k} \\
& Z_{k}=-1,698+0,020 X^{5}+0,004 X^{10}+\varepsilon_{k} \\
& Z_{k}=-0,872+0,024 X^{11}-0,085 X^{17}+\varepsilon_{k}
\end{aligned}
$$

A partir das funções discriminantes são calculados os escores $Z$ discriminantes de cada empresa (vide Tabela 5). Entretanto, para a classificação final de cada caso em um dos grupos do estudo, é necessário definir o ponto de corte (cut-off point) ou escore crítico $Z_{E C}$, que servirá para sinalizar a qual grupo pertence a empresa sob análise.

Considerando-se que as subamostras de teste apresentam números iguais de empresas em cada grupo, o ponto de corte resultará num escore crítico $Z_{E C}=0$.

Se $Z_{i}<Z_{E C} \rightarrow$ classificação no Grupo de Empresas Perdedoras

Se $Z_{i}>Z_{E C} \rightarrow$ classificação no Grupo de Empresas Vencedoras

Na Tabela 9 são apresentados, em forma matricial, os quantitativos de erros e acertos decorrentes da comparação entre o $Z_{i}$ de cada empresa e o escore crítico $Z_{E C}$.

Os resultados para os Casos Selecionados (Originais e Validação Cruzada) correspondem às classificações das empresas da subamostra de análise, isto é, após a estimação da função discriminante, o mesmo grupo de empresas é utilizado para a classificação.

A Tabela 9 mostra que as funções discriminantes estimadas nos três exercícios 
proporcionam níveis de acertos de classificações iguais ou superiores a 62,5\%, ao avaliar os escores $Z_{i}$ das empresas das subamostras de análise. Esse resultado denota, em princípio, que o uso das funções contribui para a melhoria no processo de discriminação entre grupos, quando comparado à probabilidade de classificação ao acaso, cuja expectativa seria de $50 \%$.

Tabela 9 - Matriz de classificação resultante da Análise Discriminante

\begin{tabular}{|c|c|c|c|c|c|c|c|c|c|c|c|c|}
\hline \multicolumn{4}{|c|}{ Exercícios } & \multicolumn{3}{|c|}{2005} & \multicolumn{3}{|c|}{2006} & \multicolumn{3}{|c|}{2007} \\
\hline & & \multicolumn{2}{|c|}{$\begin{array}{c}\text { Grupos } \\
\text { Reais }\end{array}$} & \multicolumn{2}{|c|}{$\begin{array}{c}\text { Grupos } \\
\text { Previstos }\end{array}$} & \multirow[t]{2}{*}{ Total } & \multicolumn{2}{|c|}{$\begin{array}{c}\text { Grupos } \\
\text { Previstos }\end{array}$} & \multirow[t]{2}{*}{ Total } & \multicolumn{2}{|c|}{$\begin{array}{l}\text { Grupos } \\
\text { Previstos }\end{array}$} & \multirow[t]{2}{*}{ Total } \\
\hline & & & & Perd. & Venc. & & Perd. & Venc. & & Perd. & Venc. & \\
\hline \multirow{8}{*}{ 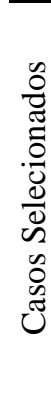 } & \multirow[t]{4}{*}{ Originais } & \multirow[t]{2}{*}{ Conta } & Perd & 24 & 16 & 40 & 33 & 5 & 38 & 29 & 11 & 40 \\
\hline & & & Ven & 14 & 26 & 40 & 21 & 17 & 38 & 17 & 23 & 40 \\
\hline & & \multirow[t]{2}{*}{$\%$} & Perd & 60,0 & 40,0 & 100,0 & 86,8 & 13,2 & 100,0 & 72,5 & 27,5 & 100,0 \\
\hline & & & Ven & 35,0 & 65,0 & 100,0 & 55,3 & 44,7 & 100,0 & 42,5 & 57,5 & 100,0 \\
\hline & \multirow{4}{*}{$\begin{array}{l}\text { Validação } \\
\text { Cruzada }\end{array}$} & \multirow[t]{2}{*}{ Conta } & Perd & 24 & 16 & 40 & - & - & - & 29 & 11 & 40 \\
\hline & & & Ven & 14 & 26 & 40 & - & - & - & 17 & 22 & 40 \\
\hline & & \multirow[t]{2}{*}{$\%$} & Perd & 60,0 & 40,0 & 100,0 & - & - & - & 72,5 & 27,5 & 100,0 \\
\hline & & & Ven & 35,0 & 65,0 & 100,0 & - & - & - & 45,0 & 55,0 & 100,0 \\
\hline \multirow{4}{*}{$\begin{array}{l}0 \\
\frac{\vec{d}}{\Delta} \\
\tilde{D} \\
i \mathbb{2} \\
z\end{array}$} & \multirow[t]{4}{*}{ Originais } & \multirow[t]{2}{*}{ Conta } & Perd & 19 & 7 & 26 & 21 & 4 & 25 & 20 & 8 & 28 \\
\hline & & & Ven & 8 & 18 & 26 & 19 & 6 & 25 & 14 & 14 & 28 \\
\hline & & \multirow[t]{2}{*}{$\%$} & Perd & 73,1 & 26,9 & 100,0 & 84,0 & 16,0 & 100,0 & 71,4 & 28,6 & 100,0 \\
\hline & & & Ven & 30,8 & 69,2 & 100,0 & 76,0 & 24,0 & 100,0 & 50,0 & 50,0 & 100,0 \\
\hline & \multicolumn{3}{|c|}{ Níveis de Acertos } & \multicolumn{3}{|c|}{$\begin{array}{l}\text { Casos selecionados } \\
\text { Originais.............62,5\% } \\
\text { Validação Cruz... 62,5\% } \\
\text { Casos não selecionados } \\
\text { Originais..............71,2\% }\end{array}$} & \multicolumn{3}{|c|}{$\frac{\text { Casos } \quad \text { selecionados }}{\text { Originais...........65,8\% }}$} & \multicolumn{3}{|c|}{$\begin{array}{l}\text { Casos selecionados } \\
\text { Originais...............65,0 } \\
\text { Validação Cruz...63,8\% } \\
\text { Casos não selecionados } \\
\text { Originais...........60,7\% }\end{array}$} \\
\hline
\end{tabular}

Fonte: Dados da pesquisa - SPSS

As classificações dos Casos Não Selecionados (Originais), por outro lado, são realizadas a partir da subamostra de teste, conferindo validade externa à função discriminante. Essas classificações podem ser consideradas de maior interesse para o estudo, pois o fato de tratar-se de um grupo de empresas não utilizado para a estimação da função discriminante, minimiza a probabilidade de um viés ascendente na precisão preditiva da função.

Os níveis de acertos nas classificações das empresas das subamostras de testes variam de $54 \%$ a $71,2 \%$. O menor nível de sucesso ocorre no ano de 2006 , mas o resultado para este ano deve ser desconsiderado, pois a premissa da homogeneidade das matrizes de variânciascovariâncias não é atendida nesse exercício social. Como se trata de uma premissa-chave da análise discriminante, um resultado mais adequado deve ser buscado pelo uso de uma metodologia que dispense tal premissa, tal como o Logit. Nos demais exercícios, 2005 e 2007, aquela premissa foi atendida e os níveis de acertos são um pouco mais expressivos.

\section{CONCLUSÕES}

Uma das primeiras constatações na aplicação das metodologias foi a de que um número bastante reduzido de índices apresentou significância estatística à diferenciação entre as empresas integrantes das amostras: em 2005, o índice Margem Bruta; em 2006, o Prazo de Recebimento e a razão Dívida Financeira/Patrimônio Líquido; e, em 2007, a razão Dívida Financeira de Curto Prazo/Dívida Financeira Total e o Grau de Alavancagem Operacional. Esse fato sinaliza que as médias dos índices das empresas não-financeiras do mercado de ações brasileiro, em ambos os grupos, apresentam perfis bastante semelhantes. Portanto, a análise conjunta de empresas dos mais diversos setores, como neste estudo, requer a inserção 
de um número substancial de variáveis, a fim de identificar aquelas consideradas relevantes ao estudo em cada exercício social.

Apesar de apresentar um reduzido número de variáveis estatisticamente significantes, os resultados da aplicação da Regra do Qui-quadrado Mínimo mostrou percentuais relevantes de acertos nas previsões de classificações em dois exercícios sociais (2005 e 2007), indicando que o uso dessa metodologia contribui para melhorar os resultados das decisões de investimentos, quando comparado às escolhas ao acaso. $\mathrm{O}$ fato de não haver homogeneidade das variâncias entre os grupos de Perdedoras e Vencedoras pode ter influenciado no percentual de acertos pouco relevante no exercício de 2006.

Para conferir mais robustez aos achados, foi aplicada a Análise Discriminante às amostras da pesquisa, obtendo-se os mesmos resultados alcançados pela metodologia da Regra do Qui-quadrado Mínimo. Portanto, considerando os resultados deste estudo, concluise que é possível aceitar a hipótese de que a AFF é capaz de prever variações de valor da empresa, contribuindo para a escolha entre melhores e piores alternativas de investimentos no mercado de ações brasileiro.

Destaca-se que não foram consideradas variáveis exógenas, tais como o PIB, taxa de câmbio, taxa de juros de mercado, delimitando a pesquisa à análise financeira por meio de índices econômico-financeiros. Os resultados da pesquisa contribuem para reflexões acerca das potencialidades das informações contábeis às escolhas entre alterantivas de investimentos, ao analisar a capacidade preditiva dos índices econômico-financeiros denominados tradicionais, e podem despertar acadêmicos e preparadores de demonstrações contábeis para a importância da aplicação de métodos econométricos nas análises das empresas.

\section{REFERÊNCIAS}

ANJOS, Vera Marleide Loureiro dos. A utilidade das demonstrações contábeis para a tomada de decisão sob a ótica dos analistas de investimentos e profissionais de relações com investidores - RI. 2008. 111 f. Dissertação (Mestrado em Ciências Contábeis) Universidade de Brasília, Brasília, 2008.

ASSAF NETO, Alexandre. Finanças corporativas e valor. 3. ed. São Paulo: Atlas, 2007.

ASSAF NETO, Alexandre; SILVA, César Augusto Tibúrcio. Administração do capital de giro. 3. ed. São Paulo: Atlas, 2002.

BESLEY, Scott; OSTERYOUNG, Jerome S. Determining trade credit default probabilities using the minimum chi-square rule. In: KIM, Young; SRINIVASAN, Venkat. Advances in working capital management. Greenwich: JAI Press., 1991. v. 2. pp. 173-195.

BETTMAN, Jenni L.; SAULT, Stephen J.; WELCH, Emma L. Fundamental and technical analysis: substitutes or compliments? 2006. Disponível em: <http://papers.ssrn.com/sol3/ papers.cfm?abstract_id=899879>. Acesso em: 1 nov. 2009. http://dx.doi.org/10.2139/ssrn.899879.

CHAN, Louis K. C.; HAMAO, Yasushi; LAKONISHOK, Josef. Fundamentals and stock returns in Japan. The Journal of Finance, v. 46, n. 5, p. 1739-1764, Dec., 1991. http://dx.doi.org/10.2307/2328571.

CLUBB, Colin; NAFFI, Mounir. The Usefulness of Book-to-Market and ROE Expectations for Explaining UK Stock Returns. Journal of Business Finance \& Accounting, n. 34, p. 132, Jan./Mar., 2007. http://dx.doi.org/10.1111/j.1468-5957.2006.00662.x

FERNÁNDEZ, Pablo; REINOSO, Laura. Shareholder value creators and shareholder value destroyers in USA. Year 2001. (2002). Disponível em: <http://papers.ssrn.com/sol3/ papers.cfm?abstract_id=298218>. Acesso em: 12 jun. 2008. 
http://dx.doi.org/10.2139/ssrn.298218.

FOSTER, George. Financial statement analysis. 2nd. ed. New Jersey: Prentice-Hall, 1986.

GASPARETTO, Valdirene. O papel da contabilidade no provimento de informações para a avaliação do desempenho empresarial. Revista Contemporênea de Contabilidade. v. 1, n. 2, p. 11-40, jul./dez. 2004.

HAIR, Joseph F. Jr et al. Análise multivariada de dados. Tradução Adonai Schlup Sant'Anna. 6. ed. Porto Alegre: Bookman, 2009.

KOTHARI, S. P. Capital markets research in accounting. Journal of Accounting and Economics, n. 31, p. 105-231, 2001. http://dx.doi.org/10.1016/S0165-4101(01)00030-1

LEMES JÚNIOR, Antônio Barbosa; RIGO, Cláudio Miessa; CHEROBIM, Ana Paula Mussi Szabo. Administração financeira: princípios, fundamentos e práticas brasileiras. Rio de Janeiro: Campus, 2002.

LOPES, Alexsandro Broedel; GALDI, Fernando Caio. Does financial statement analysis generate abnormal returns under extremely adverse conditions? 2006. Disponível em: <http://ssrn.com>. Acesso em: 12 jun. 2008.

LYRA, Ricardo Luiz Wüst Corrêa de. Análise hierárquica dos indicadores contábeis sob a óptica do desempenho empresarial. São Paulo, 2008. 171 f. Tese (Doutorado em Controladoria e Contabilidade) - Faculdade de Economia, Administração e Contabilidade da Universidade de São Paulo, São Paulo, 2008.

MYER, John N. Análise das demonstrações financeiras. Tradução de Danilo Ameixeiro Nogueira. São Paulo: Atlas, 1976.

MILTERSTEINER, Marcelo da Rosa. A validade estatística do uso de índices fundamentalistas no mercado de capitais brasileiro: um estudo aplicado ao setor bancário. Florianópolis, 2006, 85f. Dissertação (Mestrado em Engenharia da Produção) Universidade Federal de Santa Catarina, Santa Catarina, 2006.

OLSON, Dennis; MOSSMAN, Charles. Neural network forecasts of Canadian stock returns using accounting ratios. International Journal of Forecasting, n. 19, p. 453-465, 2003. http://dx.doi.org/10.1016/S0169-2070(02)00058-4

PALEPU, Krishna G.; HEALY, Paul M.; BERNARD, Victor. L. Business analysis \& valuation: using financial statements - text and cases. 3. ed. Ohio: South-Western College Publishing, 2004.

PENMAN, Stephen H. The quality of financial statements: perspectives from the recent Stock Market Bubble. Accounting Horizons, Supplement, p. 77-96, 2003. http://dx.doi.org/10.2308/acch.2003.17.s-1.77

PINTO, Juliana; HEIN, Nelson. Rough sets na análise da solvência de empresas do setor têxtil. In: ENCONTRO DA ASSOCIAÇÃO NACIONAL DE PÓS-GRADUAÇÃO E PESQUISA EM ADMINISTRAÇÃO, 32., 2008, Rio de Janeiro. Anais ... Rio de Janeiro: ANPAD, 2008. 1 CD-ROM.

PIOTROSKI, J. D. Value investing: the use of historical financial statement information to separate winners from losers. Journal of Accounting Research, v. 38, Suplement: studies on accounting information and the economics of the firm, p. 1-41, 2000. http://dx.doi.org/10.2307/2672906

QUIRIN, Jeffrey J.; BERRY, Kevin T.; O'BRYAN, David. A fundamental analysis approach to oil and gas firm valuation. Journal of Business Financial \& Accounting, v. 27, n. 7-8, 
Sep./Oct., 2000. http://dx.doi.org/10.1111/1468-5957.00335.

TATSUOKA, Maurice M. Multivariate analysis: techniques for educational and psychological research. New York: John Wiley \& Sons, 1971.

THOMSETT, Michael C. Mastering fundamental analysis: how to spot trends and pick winning stocks using fundamental analysis. Chicago: Dearborn Financial Publishing, 1998. 